\title{
Satellite-enabled educational services specification and requirements analysis based on user feedback
}

\section{Sofia Tsekeridou* and Thanassis Tiropanis}

\author{
Athens Information Technology (AIT) \\ 0.8 km Markopoulou Ave. \\ Building B7, Peania 19002, Athens, Greece \\ E-mail: sots@ait.edu.gr \\ E-mail: ttir@ait.edu.gr \\ *Corresponding author
}

\section{Dimitris Rorris and Constantinos Makropoulos}

National Center of Scientific Research 'Demokritos' (NCSR)

Athens, Greece

E-mail: dimror@dat.demokritos.gr

E-mail: cmakr@dat.demokritos.gr

\section{Tacha Serif and Lampros Stergioulas}

Department of Information Systems and Computing

Brunel University

Uxbridge, Middlesex UB8 3PH, UK

E-mail: tacha.sherif @brunel.ac.uk

E-mail: lampros.stergioulas@brunel.ac.uk

\begin{abstract}
Advanced tele-education services provision in remote geographically dispersed user communities (such as agriculture and maritime), based on the specific needs and requirements of such communities, implies significant infrastructural and broadband connectivity requirements for rich media, timely and quality-assured content delivery and interactivity. The solution to broadband access anywhere is provided by satellite-enabled communication infrastructures. This paper aims to present such satellite-based infrastructures that are capable of addressing the core requirements of rich media educational services in remote areas. The paper proceeds to examine a set of services that will realise such satellite-based distance learning systems and to assess the targeted users' interest in such services. The presented work is undertaken within the framework of the EU-funded Broadband Access Satellite Enabled Education $\left(\mathrm{BASE}^{2}\right)$ project. Furthermore, requirements analysis, based on the Volere template (Robertson) and on user feedback, is undertaken.
\end{abstract}

Keywords: satellite-enabled education; tele-education; broadband communication; service specification; requirements analysis; user feedback.

Reference to this paper should be made as follows: Tsekeridou, S., Tiropanis, T., Rorris, D., Makropoulos, C., Serif, T. and Stergioulas, L. (2008)

'Satellite-enabled educational services specification and requirements analysis based on user feedback', Int. J. Knowledge and Learning, Vol. 4, Nos. 2/3, pp.272-284. 
Biographical notes: Dr. Sofia Tsekeridou is an Assistant Professor at the Athens Information Technology (AIT) since 2006 and a member of the Software Systems and Web Applications Group. She received her Electrical and Computer Engineering diploma in 1996 and her $\mathrm{PhD}$ degree in Computer Science in 2001, both from the Aristotle University of Thessaloniki, Greece. She has published more than 50 papers in scientific journals and international conferences and has contributed to the TV Anytime and World Wide Web Consortium (W3C) standardisation bodies. Her research interests lie in the areas of multimedia processing and analysis, knowledge engineering, personalisation, information retrieval, data mining, semantic web, e-learning and gaming/edutainment.

Dr. Thanassis Tiropanis is an Assistant Professor of Software Systems and Web Applications at the AIT, where he is the Co-founder and Co-head of the Software Engineering and Web Applications Research Group. He holds a DipIng in Computer Engineering and Informatics from the University of Patras and $\mathrm{a} \mathrm{PhD}$ in Computer Science from the University of London. He teaches courses on web technologies and e-commerce. His research interests are in the areas of web technologies, semantic web, grid computing, Peer-to-Peer (P2P) systems and applications in e-commerce and e-learning. His work has been presented and published in international conferences and journals.

Dimitris Rorris holds a BSc in Physics and an MSc in 'Electronic Automation', obtained from the Computer Science Department of the University of Athens. He has remotely attended the Leonardo da Vinci programme 'eNstructor' and has been a certified e-Tutor from the University of Piraeus. He is a Certified Adult Trainer in the field of Informatics from the EKEPIS (National Certification Center) and a full member of the EPY (Greek Computer Society). He has worked in the Division of Applied Technologies of NCSR Demokritos and was involved in broadband telecommunication networks and adult training projects. He has been teaching at the Programmers' School of the Greek Ministry of Defense and at various Institutes of Adult Training of the Greek Ministry of Education.

Dr. Constantinos Makropoulos is the Director of the Division of Applied Technologies of NCSR 'Demokritos'. He is the holder of a diploma in Electrical Engineering (Energy and Telecommunications), a Masters degree in Business Economics and a Dr-Ing (PhD) degree acquired from the Technical University of Aachen, Germany, and is an Associated Professor at the same university. His main research interests include the areas of advanced communication technologies and applications (tele-education, tele-medicine). $\mathrm{He}$ has published more than 60 scientific journal and conference papers. $\mathrm{He}$ was a member of the RACE/ACTS Management Committee, the High Level Committee 'Europe and the Global Information Society' (Bangemann) and the board of the GR-NET.

Dr. Tacha Serif is a research fellow at the Department of Information Systems and Computing of Brunel University. He has been involved in a number of major EU projects, including CEBOS (FP5) and Broadband Access Satellite Enabled Education (BASE ${ }^{2}$ ) (FP6) and e-START (e-Learning). His research interests include mobile health systems, pervasive computing, wireless networks and location-based and context-aware systems.

Dr. Lampros K. Stergioulas is a Reader at Brunel University, UK, and is currently the Director of Undergraduate Studies in the Department of Information Systems and Computing of the same university. He is a qualified Chartered Engineer. $\mathrm{He}$ has published over 150 papers in journals and 
international conferences. He has supervised and examined many $\mathrm{PhD}$ dissertations in the areas of information systems, information processing, grid computing and computer science, in general. He has held numerous national and European grants in technology-enhanced learning, educational computing, human-centred information systems, communications and computing, medical and health informatics, information processing and intelligent information systems.

\section{Introduction}

The significant demands of remote geographically dispersed and isolated communities, such as agrarian and maritime, on both faster broadband access and use of advanced tele-education platforms, besides the fact that wired broadband access in these cases is always not an option, have led the research community to investigate the capabilities offered by satellite technologies in the e-learning sector. Enabling learning for members of geographically isolated communities such as agrarian, or maritime communities presents benefits in terms of promoting regional development and cost savings for governments and companies. Broadband Access Satellite Enabled Education $\left(\mathrm{BASE}^{2}\right)$ is a research project that investigates the provision of integrated synchronous and asynchronous tele-education services to remote and isolated communities through satellite and broadband wireless network infrastructures to address the needs of such communities. ${ }^{1}$

Thus, this paper initially describes satellite-based educational services designed to provide innovative and efficient tele-education solutions to geographically dispersed communities that would otherwise be excluded from the ongoing progress in the area of tele-education and e-learning. By doing so, we do not only provide faster broadband access possibilities offered by the satellite technologies to previously isolated, without any broadband access, communities, but also enable the use of innovative content and efficient tele-education platforms by these communities, so that they can actively participate in exchange of knowledge that is crucial for building a knowledge economy.

Furthermore, the purpose is to report on elicited functional and non-functional user and service requirements for content creation, distribution, management, adaptation and presentation in the context of the defined satellite-enabled tele-education services for the maritime and agricultural sectors under the capabilities offered but also the constraints imposed by the hybrid $\mathrm{BASE}^{2}$ satellite/wireless/wireline communication infrastructure.

Requirements engineering (Malan and Bredemeyer, 2001; Weiss and Araujo, 2001; Sommerville and Sawyer, 1997; Maguire and Bevan, 2002) has relied on user feedback collection based on specially designed questionnaires. A critical mass of both maritime and agrarian users has completed the questionnaire. Processing and analysis of user responses led to the generation of a requirements evaluation report and on significant feedback on specific user community interests and needs. 


\section{Satellite-based network architectures for distance learning}

Our considerations concentrated mainly on three distinct satellite network architectures for education applications, all of them being different implementations of the same generic network architecture (Figure 1). The rationale in supporting multiple satellite communication environments stems from:

- the variability of expected user requirements (some users have inflexible legacy communication systems, while others can be open for very innovative deployments)

- the existence of a number of satellite platforms with a wide range of capabilities and cost.

Figure 1 Generic satellite-based and wireless network architecture (see online version for colours)

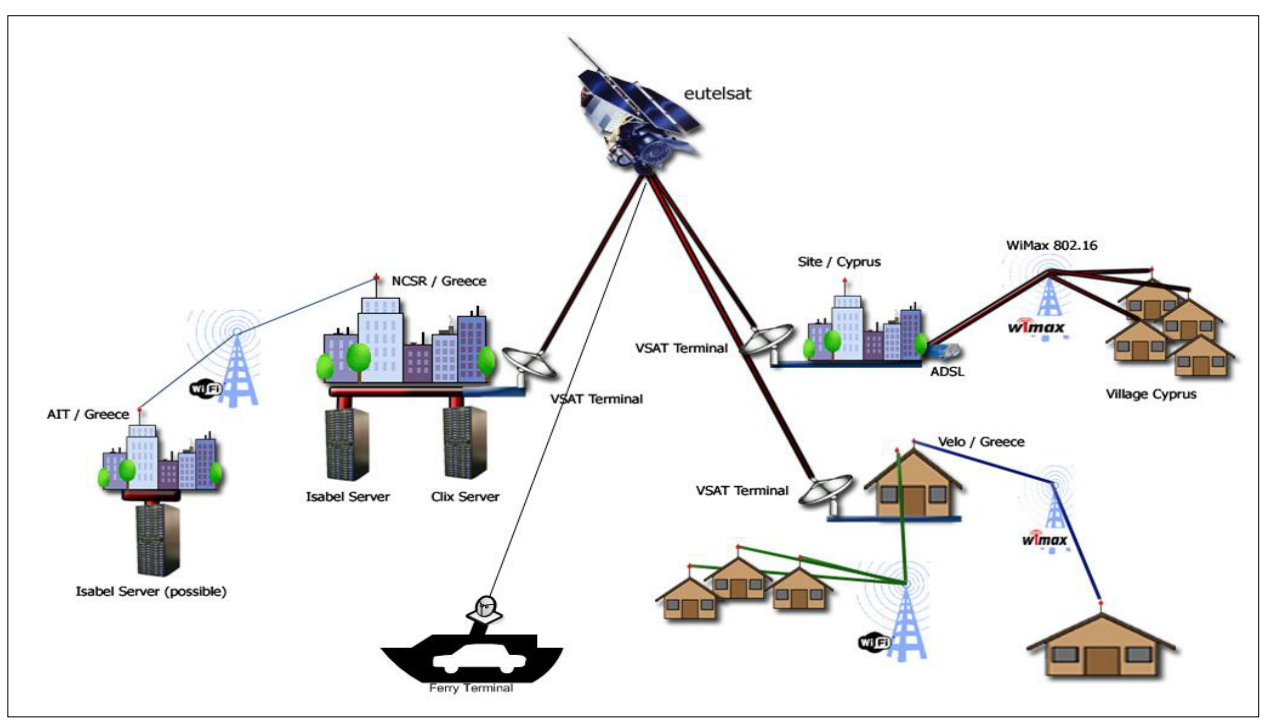

\subsection{VSAT architecture}

\subsubsection{Land-to-land VSAT architecture}

This architecture includes a number of VSAT enabled sites and further interconnection using WiFi and/or WiMax wireless infrastructure for delivering the service to more users. Different usage scenarios can involve point-to-point, point-to-multipoint interactive communication, live lectures and video on demand capabilities, as well as interconnection between the network sites in order to ensure the necessary collaboration between the learning groups.

\subsubsection{Land-to-vessel VSAT architecture}

This network architecture includes one or more ship(s) connected with a VSAT station at the central node with a fully equipped studio. It facilitates live lectures and video on demand capabilities using IPv4 protocol. In order to realise the proposed e-learning 
services on VSAT SCPC networks, we have a minimum of 500-550 ms Round Trip Time (RTT) using a 1.8-2.4 metre antenna with 4/8/16/25 Watts SSPA/TWTA. The required satellite operates in the $\mathrm{Ku}$ band approximately at $11.5 \mathrm{GHz}$ up to $8 \mathrm{Mbps}$ carrier, with a bit-error-rate equal or less than $10^{8}$.

\section{$2.2 \quad$ DVB-S/S2 architecture}

This architecture involves DVB-S or DVB-S2 enabled sites for e-learning. It can support e-learning scenarios, such as point to point and point to multipoint interactive communication and collaboration, as well as live lectures and video on demand capabilities using IPv4 and IPv6 protocols. In a DVB-S/S2 architecture, the quality of service requirements is quite different than of VSAT's. The expected RTT should be in the range of 250-280 ms unidirectional and 300-2000 ms bidirectional (with terrestrial return). The dish at the hub station should be 3.7 to 9 metres with 100+ Watts TWTA output and at the receiver end 1 to 2 metres. Depending to the technology used, network bandwidth could reach up to $120 \mathrm{Mbps}$ in the range of s1.5-36 GHz. In order to achieve a smooth e-learning content transmission the overall Bit-Error-Rate (BER) should be less than $10^{9}$.

\subsection{DVB-RCS architecture}

This architecture involves DVB-RCS enabled sites connected with the central DVB-RCS platform. Similarly to the previous architecture, this architecture can support live lectures and video on demand capabilities using IPv4 protocol. The DVB-RCS architecture follows strictly the approach of a star distribution system with one outgoing broadband satellite channel from a central hub station and multiple return channels originating from VSATs which log-in to a network management system via the satellite link. The return channel architecture is organised in MF-TDMA time slots in which either MPEG-2 or ATM cells are utilised. In order to guarantee a quality transmission of e-learning services on such infrastructure, the expected RTT should be in the range of $580-650 \mathrm{~ms}$ and overall BER less than $10^{-9}$. The dish at the hub station should be 3.7 to 9 metres with 100+ Watts TWTA output and at the remote station .98-2.4 metres with 1-4 Watt BUC SSPAs. The bandwidth for the hub station would be 1.5 to $45 \mathrm{Mbps}$ at $1.5-36 \mathrm{GHz}$, and for the remote stations .016-2048 kbps.

\section{Satellite-enabled educational services}

\subsection{Virtual classroom service}

A virtual classroom is defined as a computer accessible, online learning environment intended to fulfil many of the learning facilitation roles of a physical classroom. It provides a distributed learning environment at any time, any place and at any pace.

By using virtual classrooms, one can hold face-to-face conversations and collaborate with co-workers around the world. 


\subsection{Learning Content Management System (LCMS) service}

An LMS/LCMS supports the authoring, publication and management of learning content. Multimedia content adaptation to account for bandwidth variability and personalised access to combined Learning Objects (LOs) in a learning path that suits best the learners of the participating communities are included in the LMS/LCMS offered services.

\subsection{Tele-conferencing service}

This service enables live sessions connecting many geographically dispersed sites, either in an ad hoc or pre-arranged manner. The functionality offered by a tele-conferencing service is similar to that of the virtual classroom, however it is more focused on live interaction and more flexible with regard to time scheduling and site participation, but with limitations in terms of delivering materials.

\subsection{Webinar/Webcast service}

This service consists of a unidirectional, live or pre-recorded, transmission of content from one site to the rest of the remote sites. The date and details of each session should be announced previously in order for the receiving site to tune in properly.

\subsection{Other services}

Pre-recorded educational content is either broadcasted over satellite core and access networks (DVB-S2, VSAT) or multicasted over broadband satellite and wireless IP-based access networks (DVB-RCS, Wi-Fi, WiMAX) respectively to remote groups of learners gathered in a tele-education hall, equipped with receiving satellite antennas and hubs or wireless access points, set-top boxes or PCs, video projectors, VCRs, TV sets, microphones and speakers. The session is initiated either at pre-scheduled times or by the course coordinator and it involves, initially passive viewing of the educational course and later on communication and interaction possibilities for Q\&A sessions.

\section{User feedback evaluation}

Assessing service requirements involves extensive user feedback. This relied on a specially designed user questionnaire, including a number of sections on different envisioned services, distribution of the questionnaire to a critical mass of users from both maritime and agrarian communities from different regions, completion of the questionnaires by the users, analysis of responses. The latter provided insight into the user/service requirements for tele-education services and their desired features, and therefore assisted in the service elicitation process.

A total number of 27 maritime users and 49 agrarian users, summing up to 76 users, completed the questionnaire. Maritime users have been employees of the Superfast Ferries and the Minoan Lines companies, on ships that also undertake international routes. Agrarian users were gathered from distributed geographic locations in Greece, to 
measure differentiating user interests and needs per regions, namely Northern Greece, Peloponnesus, Mainland Greece, Greek islands. Conclusions drawn per service and for the integrated services platform are summarised below.

\subsection{Users profile summary}

Approximately $76,32 \%$ of the users were men and $23,68 \%$ were women. Half of them were in the 35-49 range of age, while the rest were mainly younger $(>18)$, and almost all of them were employed. The majority of those attend educational seminars or programmes only a few times a year or seldom. The majority of users are accustomed to technology and electronic communication.

\subsection{User interest in synchronous e-learning/tele-education services}

Synchronous services refer to synchronous communication and collaboration among users and tutors.

\subsubsection{Virtual classroom service}

Currently the members of both communities do not get the chance to participate in training programmes very often; training in the maritime community presents the lower frequency according to the questionnaire results. The percentages for the agrarian community are higher. Nevertheless, both communities seem eager to participate in virtual classrooms (more than $85 \%$ in total), while around $90 \%$ of both community members have never had the chance to do so in the past.

Both communities show a slight preference to be able to participate in virtual classes from home $(60 \%$ on average) with the agrarian community showing a slightly higher preference in this, as seen in the chart below.

Almost $88 \%$ of the total of both community members would like to be able to interact during virtual classroom sessions not only with the tutor but with the other participants.

\subsubsection{Tele-conferencing service}

Almost $90 \%$ of the members of both communities have never participated in a teleconference. Nevertheless, $54 \%$ of the participants in this survey are very or extremely interested in participating with colleagues from remote areas in future, while a significant $32 \%$ appears to be open to this possibility.

Approximately 2 in 3 of the participants are interested in communicating with tutors based on remote locations by teleconferencing; the interest for this feature is stronger in the agrarian community. In addition, while the agrarian community appears to be equally interested in the whole spectrum of learning activities via teleconferencing (collaboration with colleagues, collaborative learning, seeking tutor advice, exchange of know how) the maritime community appears to be less interested in collaborative learning or interaction with tutors than in exchange of know-how and collaboration with colleagues.

Approximately 1 in 4 of the participants consider it very or extremely important to be able to use TV-based equipment instead of a PC for participating in video conferences as shown in the chart below. Approximately 1 in 5 could either use a PC or TV-based equipment. 
Half of the participants consider very or extremely important the following: flexibility for creating, advertising and scheduling video conferences, one-click access to video conferencing services, video communication, file sharing, chatting application.

\subsubsection{Webinar/Webcast service}

Almost none of the participants have had the experience of participating in a Webinar/Webcast session. However, half of them are very or extremely interested in participating in Webinars, while more than 1 in 3 say they are open to this potential.

It seems that the intended use of Webinars is primarily for collaboration with peers and collaborative learning with peers.

About $70 \%$ of the maritime community participants favour participation from home, while the agrarian community is almost equally split between participants who prefer home-based and those who prefer classroom-based Webinars.

Based on the results of this survey, 1 in 3 of the members of the maritime community consider very or extremely important to be able to participate in Webinars using TV-based equipment, while one 1 in 4 members of the agrarian community place high importance on this feature.

\subsection{User interest in asynchronous e-learning/tele-education services}

Asynchronous services refer to mainly individual user access to learning resources and non-synchronous communication with other users or tutors.

\subsubsection{Learning (content) management system service}

For both communities 3 out of 4 have never yet used a Learning Management System (LMS) and an educational portal to access education resources. However, around $60 \%$ of both communities is eager to use such a system.

Half of the users of both communities would use the LMS/LCMS system for collaboration with other colleagues and exchange of ideas. The second strongest reason is the ability to easily access educational resources and receive feedback from experienced tutors at a $41 \%$. The timely updating of recent policies in their professional interests and the personalisation features of the learning process follow in their interest.

An average of $60 \%$ of the users prefers access from home than from a classroom, with maritime users showing an increased interest for this option.

In terms of LMS service features (suggested list of educational courses based on user interests, personalised presentation style, preferred data formats, personal guidance, self-assessment and resume capability), the most preferred one is the first one, while the personalised presentation style of educational content is second in favour. The maritime community is more interested in advanced capabilities than the agrarian community.

Since personalised services involve user profile data management and storage, as well as user behaviour modelling, users were asked on how comfortable they would feel in such cases. More than 1 out of 4 would feel comfortable in both cases, while almost half of the users would be interested in experimenting in such situations.

A $62 \%$ of users in average believe the communication with remote tutors and learners is of great importance. The preferred modes of remote communication and collaboration are e-mails (64\%), chat (50\%) and forums (44\%). 
To the option to receive low quality video streaming of educational lectures but in timely manner rather than high quality videos but with longer delays, users from both communities are open to adapted implementations per case.

Finally, both communities are in favour of the dynamic adaptation feature of the learning process, believing in its efficiency to provide enhanced learning experiences.

\subsubsection{Other services}

More than $40 \%$ of the users of both communities are very or extremely interested in passively attending educational courses in tele-education rooms, while more than $30 \%$ of the users are open to such a possibility. There is however a number of users that are interested in more advanced tele-education services.

The interest of both communities in using both types of scenarios of this service, that of passively viewing scheduled educational programmes at tele-education halls and that of participating to an afterward Q\&A sessions with remote tutors, is almost equal. With respect to the features of such services, among media presentation devices such as TV sets, video walls, PC screens or a combination of those, the preferred devices for both communities are PC screens and video walls.

\subsection{User interest in integrated BASE ${ }^{2}$ e-learning/tele-education services}

In this case, all individual services are offered through the integrated $\mathrm{BASE}^{2}$ platform to users to select and use.

Seventy-two percent of both communities prefer synchronous services, while $28 \%$ asynchronous ones. With respect to the option of being provided with integrated services, combining both synchronous and asynchronous types of services, more than half of the users of both communities believe it very or extremely important.

\section{Generic service requirements definition}

\subsection{Project drivers}

\subsubsection{The purpose of the BASE ${ }^{2}$ service architecture}

The $\mathrm{BASE}^{2}$ service architecture will provide educational resources to selected user groups through broadband access satellite networks. The proposed service architecture supports two major objectives:

- deploying and operating educational content generation, management and delivery systems for different modes of learning over different network technologies

- providing end-to-end integrated tele-education services and applications that can be delivered to a large number of sites and implement a variety of tele-education modes.

\subsubsection{Client, customer and other stakeholders}

For the scope of the time limited $\mathrm{BASE}^{2}$ project the customers addressed come from two important economical sectors: agriculture and maritime. These communities have been selected due to their significant economical magnitude while their differences constitute a 
diverse application environment for our purpose. This way the BASE ${ }^{2}$ project has the opportunity to extract useful data for the design of a sustainable business model for a European wide satellite enabled educational system that can support a diversity of users.

The customers of the $\mathrm{BASE}^{2}$ service architecture are the individuals and companies that need e-learning services in order to get training and at the same time have expensive or unavailable terrestrial network access to get the desired services.

\subsubsection{Users of the BASE ${ }^{2}$ service architecture (actors)}

All users of e-learning services apply to our architecture approach provided they take advantage of satellite coverage to use the services.

\subsection{Functional requirements}

\subsubsection{The scope of the BASE ${ }^{2}$ service architecture}

The main purpose of the $\mathrm{BASE}^{2}$ service architecture is to facilitate the sustainable provision of integrated tele-education services and applications to a large number of distributed interconnected sites over a variety of telecommunication infrastructures. The realisation of this framework requires the seamless integration of low-cost satellite telecommunications infrastructures with wireless broadband systems.

\subsubsection{Functional and data requirements}

The major functional requirements are identified:

- The proposed network shall allow unicast, multicast and broadcast distributions.

- One or more sites should be able to transmit live or pre-recorded content to one or more of the other remote sites.

- The services provided should provide users with timely and high quality multimedia content presentations.

- The proposed architecture should support the feature of content adaptation based on the bandwidth and networking resources of remote sites and users.

- $\quad$ The $\mathrm{BASE}^{2}$ service architecture should support unidirectional and bidirectional sessions among remote sites.

- $\quad$ The BASE ${ }^{2}$ services should be integrated with the LMS.

- The LMS should share the user credentials securely among the system services.

- The LMS should provide a learning content authoring environment for course/learning object designers and tutors.

The major data requirements are: personal data (user profile information, assessment results and learning path) should be secured and preferably encrypted while it is transferred. 


\subsection{Non-functional requirements}

The major non-functional requirements are identified:

1 Look and feel requirements

- High quality multimedia need to be employed to motivate learners from targeted communities.

- Uniformity in GUI design will avoid user frustration.

2 Usability requirements

- All services must offer guidance/help based on the user profile and skills.

- All services should be easy to use and easily accessible by non-expert users.

- The usability of all services/systems should ideally be monitored and evaluated.

3 Performance requirements

- The system/services response should be fast enough to avoid interrupting the users' flow of thought.

- Asynchronous services should be robust. Synchronous ones should enable the best possible interaction among users with high-quality multimedia and optimum QoS.

4 Operational requirements

- Access to services will be achieved either from desktops in tele-education halls or from desktops at the home of the users with internet connection

- Multimedia enabled desktops will be needed (soundcard, speakers, camera/microphone).

\section{Evaluation outcome}

After such an assessment and evaluation process, a number of tele-education and e-learning scenarios were defined to present the direct deployment of $\mathrm{BASE}^{2}$ services. Each scenario may involve the deployment of part of a service, of a full service or of a combination of services based on the feedback received during the evaluation. This is the reason that services and scenarios are distinguished. Further, the specified scenarios present the basis for $\mathrm{BASE}^{2}$ trials definition and evaluation of $\mathrm{BASE}^{2}$ implemented services and infrastructure.

The scenarios are grouped into two major categories as illustrated in Figure 2:

1 The common room oriented e-learning scenarios. These address cases where groups of learners are gathered in a common room to be educated using a $\mathrm{BASE}^{2}$ service. The key factor here is groups of learners in a common room - that is, a tele-education room is setup with all required equipment to access the BASE ${ }^{2}$ service and access is mainly scheduled and occurs simultaneously. 
2 The home oriented e-learning scenarios. These refer to cases where each learner accesses the $\mathrm{BASE}^{2}$ services from home and from his own enabling equipment at any time of the day.

Figure 2 High level use case diagrams for (a) common room and (b) home based learning

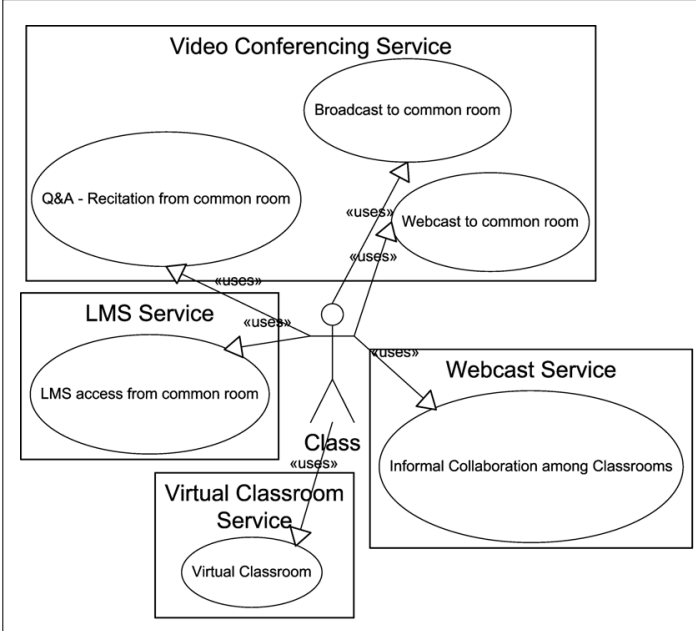

(a)

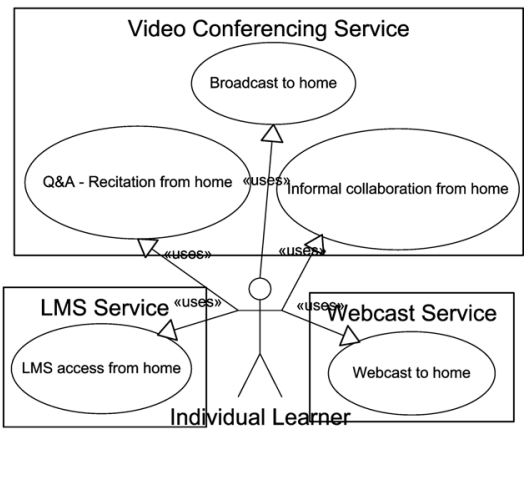

(b)

\section{Conclusions}

The educational needs of specific remote professional communities for satellite-based tele-education have been the basis for the presented work. In particular, we considered these needs in the context of providing tele-training services to professional communities that are distributed in remote inaccessible areas. Generic satellite-based service requirements of rural communities have been identified, and on this basis, three network architectures have been considered and described. Furthermore, a number of services have been identified and user feedback as to their specific needs and their interest in using these services has been investigated and processed to lead to service requirements specification using the Volere template (Robertson, 2005). Users from both communities are interested in using advanced e-learning/tele-education services to be timely updated on new policies and guidelines, collaborate with other remote colleagues or tutors and expand in this way their expertise and knowledge. They believe of high importance features like communication and personalisation/adaptation of content to their interests. They are in favour of being provided with integrated synchronous and asynchronous services. This result led to viable e-learning scenarios definitions composed of a set of synchronous and asynchronous services.

The paper makes a strong case for the development and wider adoption of satellite-enabled services, as this will extend the benefits of ICT-based education to an even larger proportion of the workforce worldwide. 


\section{Acknowledgement}

The presented work is supported by the Aeronautics and Space Programme of European Commission, in the framework of the $\mathrm{BASE}^{2}$ project (Contract no. 516159).

\section{References}

Maguire, M. and Bevan, N. (2002) 'User requirements analysis: a review of supporting methods', IFIP 17th World Computer Congress, Kluwer Academic Publishers, pp.133-148.

Malan, R. and Bredemeyer, D. (2001) 'Defining non-functional requirements', Bredemeyer Consulting, http://www.bredemeyer.com/pdf_files/NonFunctReq.pdf.

Robertson, J.S. (2005) 'Volere: requirements specification template', 10th ed., Atlantic Systems Guild, http://www.systemsguild.com/GuildSite/Robs/Template.html.

Sommerville, I. and Sawyer, P. (1997) 'Viewpoints: principles, problems \& a practical approach to requirements engineering', Annals of Software Engineering, Vol. 3, pp.101-130.

Weiss, M. and Araujo, I. (2001) 'Patterns and non-functional requirements: an interim report', MICON, http://www.scs.carleton.ca/ weiss/papers/micon-2001.pdf.

\section{Note}

1 The BASE ${ }^{2}$ Project website, http://www.dat.demokritos.gr/base2/. 